Revista

\title{
Multi-Ensayos
}

Vol. 8, núm. 15

ISSN: 2412-3285

https://multiensayos.unan.edu.ni

DOI: https://doi.org/10.5377/multiensayos.v8i15.13193

\section{Emprendimientos e innovación, una oportunidad hacia la competitividad de las Mipymes}

\section{Entrepreneurship and innovation, an opportunity towards the competitiveness of Mipymes}

Arlen Meryfel Picado Juárez ${ }^{1}$

Recibido: 26 de agosto de 2021. Aceptado: 15 de diciembre de 2021

\section{RESUMEN}

La cantidad de emprendimientos en Nicaragua han aumentado significativamente, estos son fuentes generadoras de empleo, negocios familiares que deciden emprender como un medio de subsistencia. El presente ensayo es de investigación sobre una temática de interés "emprendimiento e innovación". Se presenta una conceptualización general, datos del diagnóstico del sistema nacional de innovación en Nicaragua (SNIN), algunos estudios en diferentes sectores donde se evidencian algunas debilidades, también diversos programas de apoyo a las Mipymes, la experiencia personal de la autora de este ensayo y una propuesta de acciones para fortalecer estos negocios. La revisión bibliográfica con respecto a la temática de innovación y emprendimiento, se realizó por medio de revisión bibliográfica en artículos científicos, informes y manuales, también tesis de licenciatura, maestría y doctorado. Las investigaciones recopiladas muestran problemáticas y debilidades existentes en los diferentes sectores económicos, como la inexistente aplicación de la innovación en sus procesos productivos, poco acceso a fuentes financiamiento, baja productividad y competitividad, predominio procesos manuales y bajos niveles tecnológicos para la producción, lo que limita el crecimiento y desarrollo empresarial, provocando el estancamiento de las empresas. Es importante destacar que implementación de la ciencia, la tecnología y la innovación (CTI) en los emprendimientos contribuirá al crecimiento económico, elevar la competitividad empresarial en el corto, mediano y largo plazo, también a incrementar el nivel de vida de los empresarios y a enfrentar los desafíos con nuevos conocimientos, todo esto permitirá a Nicaragua alcanzar crecimiento económico, disminuir la pobreza y la desigualdad en los sectores económicos y sociales.

Palabras clave: emprendimiento; innovación; Mipymes.

1 Docente de la Facultad Regional Multidisciplinaria, Estelí. UNAN-Managua/FAREM-Esteli. Correo electrónico: arlenmery@ yahoo.com. ORCID: https://orcid.org/0000-0002-8817-7841

(c) 2022 Revista Multi-Ensayos. 


\section{ABSTRACT}

The number of enterprises in Nicaragua has increased significantly. Entrepreneurship is a source of employment, created by families that decide to undertake as a means of subsistence. This essay explores a topic of interest "entrepreneurship and innovation." We present a general conceptualization, data of the diagnosis of the national innovation system in Nicaragua (SNIN), some studies in different sectors where some weaknesses are evident. It also describes various programs to support Mipymes, the personal experience of the author, and a proposal for actions to strengthen these businesses. This essay reviews bibliography on innovation and entrepreneurship using scientific articles, reports, and manuals, as well as bachelor, master, and doctorate thesis. The research collected existing problems and weaknesses in the different economic sectors, such as the non-existent application of innovation in productive processes, little access to funding, low productivity and competitiveness, predominance of manual processes, and low technological levels of production. All these factors limited business' growth and development, causing companies to stagnate. It is important to emphasize, that the implementation of science, technology, and innovation (CTI) in enterprises contributes to their economic growth, raise their competitiveness in the short, medium, and long term. It also increases the standard of living of entrepreneurs, and face challenges with new knowledge. All this will enable Nicaragua to achieve economic growth, reduce poverty and inequality in the economic and social sectors.

Keywords: entrepreneurship; innovation; Mipymes.

\section{INTRODUCCIÓN}

Los emprendimientos son una fuente generadora de empleo y de producción para cualquier país. La mayoría de los emprendimientos son negocios familiares que aspiran a posicionarse en el mercado y ser competitivos en el sector que atienden.

Los emprendimientos son nuevas empresas que inician las personas por cuenta propia, con el propósito de percibir ingresos a través de la oferta de productos o servicios en diversos mercados, operando en ambiente de certeza (conocimiento y seguridad) o incertidumbre (no se conoce con exactitud una situación), asumiendo riesgos e identificando nuevas oportunidades, estos emprendimientos son llamados y conocidos como empresarios. (Herrera Guerra \& Montoya Restrepo, 2013)

Los emprendedores presentan una serie de particularidades, la más representativa es la inteligencia creativa que les permite llegar a ser promotores de cambio, por medio de la creación de proyectos de modelo de negocios, reflejando así la relación entre emprendimiento e innovación. (Liévano, Vargas, Pico, \& Pérez, 2018)

Entre algunas de las capacidades de los emprendedores esta la innovación y la gestión de sus negocios o empresas, elementos indispensables para que puedan alcanzar una ventaja competitiva en el mercado. Una vez logrado, será necesario la implementación de estrategias con actividades diferentes a la de la competencia, con el fin de estabilizarse y ser competitivos en el sector que atienden. (Liévano, Vargas, Pico , \& Pérez, 2018) 
La innovación en las Mipymes es indispensable, ya que permite la introducción de nuevos o mejorados productos o servicios, donde el cliente tiene una variedad de alternativas para elegir, la OCDE citado por Liévano, Vargas, Pico \& Pérez (2018) define: "La innovación como la introducción de un nuevo o significativamente mejorado producto -ya sea bien o servicio, un proceso, o un método de comercialización u organizativo." (pág. 307)

La empresa que innova contribuye al fortalecimiento, crecimiento y desarrollo de sus negocios y por consiguiente a las naciones en un mediano y largo plazo, mejorando el nivel de vida de sus habitantes y la competitividad de los emprendimientos. (Liévano, Vargas, Pico , \& Pérez, 2018)

El ensayo está estructurado con una variedad de definiciones que aclaran los términos de emprendimiento, innovación y la relación entre ambos, también acerca de la competitividad y los determinantes. Otros aspectos importantes es la ciencia, la tecnología y la innovación (CTI) y los datos sobre el del diagnóstico del sistema nacional de innovación en Nicaragua (SNIN). Se evidencia en los resultados de algunos estudios recopilados, las debilidades en varios sectores económicos, así como los programas que han apoyado a Nicaragua en el emprendimiento.

Se presenta la experiencia de la autora del ensayo en el ámbito educativo y en investigaciones. De igual manera, se realiza una propuesta de acciones de autoría propia para fortalecer los emprendimientos, estas, propuestas a partir de las debilidades analizadas desde los trabajos investigativos, así como del, diagnóstico del sistema nacional de innovación en Nicaragua (SNIN).

Para concluir, es oportuno enfatizar que la innovación es un reto para los propietarios de estos emprendimientos, es fundamental revisar los procesos productivos, la calidad del bien o servicio, capacitar al capital humano, la implementación de tecnología en los procesos, para obtener una posición competitiva con respecto a la competencia. Por tal razón, es de vital importancia la implementación de la CTI en estas empresas, para poder enfrentar los retos con conocimientos y tecnología para mejorar la calidad de vida de los propietarios y de los habitantes a nivel de país, dando solución a los problemas que preocupan al estado nicaragüense.

\section{DESARROLLO}

Los emprendimientos son, pequeñas empresas que surgen para resolver necesidades actuales, debido a diversas problemáticas, como el desempleo, así como también para establecer un negocio como medio de subsistencia. Estos negocios, indistintamente del motivo por el cual inician, buscan obtener ganancias y la supervivencia en el sector en el cual atienden. Con características similares como: soñar en grande, la persistencia, asumir riesgos día a día, ser generadores de empleos y llegar a satisfacer las necesidades de un sector que esta desatendido o en auge, entre otras.

El propietario de los emprendimientos es conocido como emprendedor, definido por OCDE (Ahmad \& Hoffmann, 2008), Wennekers \& Thurik, 1999 a como se citó en Somarriba Sandoval (2015) afirman que: 
Las personas que trabaja por cuenta propia, creando nuevas empresas para generar ingresos desde cualquier actividad económica mediante la oferta de productos o servicios en contextos de incertidumbre, tomando decisiones, identificando oportunidades tomando riesgos, todo emprendedor es llamado y conocido como empresario. (pág. 35)

Castillo, (1999) y; González et al., (2010), citado en Herrera y Montoya (2013), definen emprendimiento como: "El término usado para identificar a quien comenzaba una empresa, y fue ligado a empresarios innovadores" (pág. 19).

En cuanto a la cantidad de emprendimientos a nivel nacional, Managua, Estelí y Masaya son los más representativos, al respecto Urcuyo (2012) proporciona los siguientes datos:

Estelí, por ejemplo, se ubica como líder nacional en términos de emprendimiento total (incluyendo microempresas), por encima de Managua con 368 empresas por 10,000 habitantes. Managua está relegada a un segundo puesto, apenas por encima de León. Sin embargo, en términos de PYME, Managua retoma el liderazgo con 24.3 PYMES por cada 10,000 habitantes, seguido de Estelí y Masaya en ese orden. A nivel nacional el promedio es de 12.8 PYMES por cada 10,000 habitantes. (pág. 7)

Existe una estrecha relación entre el emprendimiento e innovación, Dyer, Gregersen y Christensen (2009), citado en Liévano, Vargas, Pico, Pérez (2018), "analizaron la relación que existe entre emprendimiento e innovación, donde establecen que, "los emprendedores innovadores cuentan con una inteligencia creativa que les permite ser agentes de cambio en la generación de proyectos de modelo de negocio exitosos" (pág. 307).

La Organización de Cooperación y Desarrollo Económicos (ODCE) (2005) define:

Una innovación es la introducción de un nuevo, o significativamente mejorado, producto (bien o servicio), de un proceso, de un nuevo método de comercialización o de un nuevo método organizativo, en las prácticas internas de la empresa, la organización del lugar de trabajo o las relaciones exteriores. (pág. 56)

Emprender es crear empresas, las que contribuyen a incrementar el nivel socioeconómico de los países por que se generan ingresos, la disminución de la pobreza y el bienestar de sus ciudadanos, según Santillán, Gaona \& Hernández, (2015), citado en Liévano et, al. (2018). Al iniciar una empresa, es importante tomar en cuenta las oportunidades de innovación y experimentar ideas propias con flexibilidad y mente abierta, aquí se hace necesario pensar en el crecimiento económico basado en la innovación de negocios, según Pico et al., (2010) como fue citado en Liévano et, al. (2018).

Uno de los aliados para el crecimiento de las economías nacionales es el emprendimiento innovador, para los países europeos, así como en América Latina y Estados Unidos, en esta era de globalización, la competitividad es fuerte y se hace necesario que las empresas creen valor, utilizando la innovación, elemento fundamental para alcanzarlo, (Liévano et, al. (2018)). 
Los emprendedores tienen la capacidad de innovar y gestionar sus empresas, aspectos importantes para obtener una ventaja competitiva, al lograrlo, se deberá implementar diversas estrategias por medio de una variedad de actividades distintas a la de la competencia, para mantenerse en competitividad en el sector, (Díaz, 2009, Porter, 1990 citado en Liévano et, al. (2018))

Al respecto del planteamiento, la innovación es uno de los factores claves para consolidar el ambiente competitivo empresarial, adquirir esas ventajas y obtener beneficios en sus actividades requiere del compromiso de conducir hacia procesos de aprendizaje organizacional, con el propósito de transferir y crear conocimientos. De igual manera, el uso de tecnologías en empresas emprendedoras a nivel mundial, para poder ubicarse en un mercado competitivo, juega un papel significativo. (Ahumada \& Perusquia, 2015, Mendoza \& Valenzuela, 2013, Ríos \& Marroquín, 2012, citado en Liévano et, al. (2018))

La competitividad se traduce en el beneficio sostenible para la empresa o institución, también como el resultado o la mejora de calidad permanente acompañada de la innovación, (Koontz y O’Donnell, 2013, citado en Hernández (2016)).

Sallenave (2014), citado en Hernández (2016) brinda otra concepción de competitividad:

Hace referencia a la capacidad que tiene una empresa o país de obtener rentabilidad en el mercado en relación a sus competidores. Esta depende de la relación entre el valor y la cantidad de producto ofrecido y los insumos necesarios para obtenerlos (productividad) y a productividad de los otros oferentes del mercado. (pág. 4)

De igual manera, la competitividad también tiene incidencia desde la forma de plantear y desarrollar iniciativas de negocios, esto ha marcado la transformación en los modelos de empresa y empresario, (Jiménez Coronado, 2016).

Molina y Sánchez (2016) enlistan los principales determinantes de la competitividad:

Se enuncian: la constante innovación; la productividad en las operaciones de la empresa; la calidad del bien o servicio; el énfasis para el desarrollo del capital humano; la implementación tecnológica en los procesos junto a las actividades de investigación y desarrollo (I+D); y la correcta gestión financiera para evitar potenciales quiebras. (pág. 107)

Otro aspecto muy importante a abordar es el de la ciencia, la tecnología y la innovación (CTI), reconocidas como motores centrales de crecimiento económico de mediano y largo plazo. Las innovaciones tecnológicas permiten un incremento del nivel de vida de los individuos y en una mayor competitividad de las empresas, al igual que de los países. De igual manera, "permite la incorporación a la economía del conocimiento y elevar la competitividad de los sectores estratégicos, la CTI le proporcionará a Nicaragua la oportunidad de conseguir un crecimiento económico, disminuir la pobreza y la desigualdad." (CONICYT Presidencia de la República, 2010, pág. 6).

Entre otros aspectos que permitirán la CTI al país están: "el enfrentar los desafíos, con nuevos conocimientos y nuevas aplicaciones tecnológicas que facilitaran a optimizar la calidad de vida de los nicaragüenses, por 
ende, a resolver problemas en áreas de preocupación estratégica, como la competitividad industrial, el uso y generación de energía, la salud y el medio ambiente", (CONICYT Presidencia de la República, 2010, pág. 21)

Un dato importante a destacar es el del diagnóstico del sistema nacional de innovación en Nicaragua (SNIN), que reveló que existen capacidades para usar, mejorar y generar ciencia, tecnología e innovación en los diversos actores del sistema, pero al igual se detectaron debilidades, tales como:

- Una industria formada principalmente por micro y pequeñas empresas, generalmente caracterizadas por tener baja productividad y baja competitividad. Predominan los procesos manuales y bajos niveles tecnológicos, la escasa inversión en actividades de investigación y desarrollo, y la reducida presencia de innovaciones de productos y de procesos.

- Los centros públicos de investigación enfrentan restricciones significativas para sus actividades debido a la insuficiente disponibilidad de recursos humanos y financieros, procesos inadecuados de reclutamiento de investigadores, falta de infraestructura y la persistencia de bajas remuneraciones.

- Las organizaciones públicas encargadas de fomentar la CTI se enfrentan con escasos recursos para financiar sus programas $y$, en ocasiones, para contar con una plantilla laboral que les permita llevar a cabo políticas de largo alcance.

- La oferta de programas educativos a todos los niveles es insuficiente para cubrir las necesidades prioritarias del país y los programas profesionales carecen de actualización.

- La falta de recursos y la reducida actividad de los investigadores, dedicados principalmente a la docencia, son las causas de la deficiente calidad y pertinencia de la investigación en las universidades.

- Las relaciones entre universidades y empresas para intercambiar, mejorar o crear conocimientos científicos y tecnológicos son débiles.

- Escasez de recursos para financiar proyectos productivos empresariales y más aún para actividades de innovación. (CONICYT Presidencia de la República, 2010, pág. 6)

A continuación, se presentan algunos estudios, en diferentes sectores donde se evidencian algunas de las debilidades antes mencionadas:

En el sector servicio de comida, en la investigación presentada por Picado Juárez (2016), los principales hallazgos están que: Los propietarios implementan 8 de los 9 factores determinantes de la competitividad, a excepción del quinto factor; innovación, los propietarios indicaron que ellos varían el menú diariamente y semanalmente, lo cual no es propiamente innovar, la teoría plantea que el éxito de las empresas también se asocia al desarrollo de nuevos productos. Los cambios e innovación con lleva a ofertar nuevas propuestas para los clientes. En este tipo de negocio es de mucha importancia la variedad en el menú, la innovación en cuanto a la oferta de platillos, la presentación de los mismos, la decoración del local, respetando siempre las normas del buen gusto y lo que para sus clientes es cómodo.

En el sector tortillero, estudio presentado por Díaz, Espinoza y Mairena (2017), los autores determinaron que el 91 \% de las encuestadas producen su producto de manera manual, artesanal y desfasada. A pesar de tener ideas y deseos, el $85 \%$ han pensado en desarrollar un proceso de producción mecanizada y/o automatizado, y sea por falta de conocimiento en la mayoría de los casos, las herramientas para 
la producción las ven como un gasto o no cuentan con el capital o financiamiento para mecanizar o automatizar su proceso de producción.

En el sector tabaco, estudio presentado por Centeno, Gámez y Mendoza (2020), se concluye que se destacó la importancia de innovar por parte de los colaboradores, pero existe una debilidad en la misma ya que no cuenta con una evolución continua en la tecnología.

El sector hortícola de la papa en el Norte de Nicaragua, en el estudio presentado por Picado Vanegas (2016), se concluye que el sector tiene limitada disponibilidad de tecnologías y del accionar de los actores claves en los procesos de generación y transferencia de tecnologías; esto, está propiciando reducido desarrollo de las innovaciones, baja productividad y acelerado el impacto ambiental. Las innovaciones de producto y comercialización son casi inexistentes. Las innovaciones de proceso y organizativas, son limitadas.

Nicaragua ha contado con diversos programas enfocados en las Mipymes, desde el año 2002 se ejecutó el proyecto de apoyo a la innovación tecnológica (PAIT), del Ministerio de Fomento, Industria y Comercio (MIFIC), producto de un préstamo del Banco Interamericano de Desarrollo (BID). Dio financiamiento a Mipymes para desarrollar proyectos de innovación tecnológica.

De igual manera, el Instituto Nacional Tecnológico (INATEC) desarrolla programas estratégicos articulados con el Ministerio de Economía Familiar Cooperativo y Asociativo (MEFCCA), con el fin de llevar la capacitación a los protagonistas de las Mipymes en los sectores productivos de: Comercio y Servicio, Agropecuario y Forestal e Industria y Construcción.

Para el año 2004, se dio la participación de las universidades que conforman el Consejo Nacional de Universidades (CNU) en Nicaragua, con el programa "La Universidad emprendedora", orientado a desarrollar el papel y la contribución de las Universidades en los procesos de innovación. Este programa dio la oportunidad a las universidades a compartir sus experiencias, competencias, estrategias y actividades, así como, con otros actores claves en los sistemas de innovación.

El MEFFCA ejecuta diversos programas, entre los que se mencionan: El programa para el desarrollo de la cadena productiva del bambú, programa para el desarrollo del sector porcino, programa socio productivo patio saludable, programa de microcréditos para emprendimientos en el campo, entre otros.

Actualmente el programa de Nicaragua Creativa ejecuta los programas de: Economía creativa, Prospera, Nicaragua emprende, entre otros.

El ente regulador en el ámbito de la ciencia y tecnología en los diferentes sectores del país, es el Consejo Nicaragüense de Ciencia y tecnología (CONICYT), quien coordina los programas, proyectos y actividades científico -tecnológicos, de igual manera sirve de enlace entre organismos nacionales e internacionales con los actores de ciencia y tecnología de Nicaragua. 
En Nicaragua, actualmente existe una variedad de lineamientos y estrategias encaminados a la mejora en sectores estratégicos, desglosados en el Plan Nacional de Desarrollo Humano (PNDH, 2018-2021), donde uno de los sectores claves por su representatividad y relación con los demás sectores de la economía, es el sector de la micro, pequeña y mediana empresa (Mipymes).

En relación a la temática desarrollada y la experiencia personal de la autora de este ensayo, esta se ha visto reflejada desde su labor docente en la Facultad Regional Multidisciplinaria (FAREM-Estelí, UNANManagua). De igual manera, ha brindado capacitaciones a pequeños empresarios de la ciudad de Estelí, organizadas por el Instituto Nicaragüense de Turismo (INTUR), en temáticas de Administración, Relaciones humanas, Atención al cliente, entre otros temas.

Otra experiencia a mencionar, es realización de investigaciones a nivel de licenciatura y maestría, estudiando los sectores de alimentos y el de procesamiento de lácteos, donde se visibilizan problemáticas generalizadas en los sectores, en cuanto a: carencia de tecnología e innovación en sus procesos, de asociatividad, en el manejo administrativo y contable, realizándolo de forma empírica en cuanto a la visión empresarial, entre otros.

Posterior al haber planteado las dificultades y debilidades que enfrentan los emprendedores en el tema de la innovación, identificadas desde los trabajos investigativos, así como en el diagnóstico del (SNIN), es oportuno realizar una propuesta de acciones de autoría propia, que permitirán fortalecer los negocios de los emprendedores:

- Realizar un diagnóstico de los emprendimientos, para determinar las fortalezas y debilidades, esto, para identificar las necesidades y demandas reales del sector.

- Brindar capacitación y asistencia técnica en temáticas de interés, a los propietarios y al personal de los emprendimientos de forma permanente.

- Asociarse con otros emprendimientos para fortalecer los lazos de cooperación y colaboración.

- Los emprendedores deben de abocarse a las instituciones (CONICYT, MEFCCA, INATEC, entre otros) para recibir el apoyo que ofrecen a los emprendedores.

- A las autoridades municipales y académicas impulsar en las Mipymes la mejora continua y adaptación de tecnología en los procesos de manufactura para potenciar y aumentar la productividad con el objetivo de hacerlas competitivas e ingresarlas en las cadenas de valor nacional e internacional.

- Fortalecer el vínculo entre Universidad y los Centros de investigación con las Mipymes, para desarrollar procesos productivos innovadores que satisfagan las necesidades de la población.

- Sistematizar y divulgar los casos exitosos de Mipymes industriales que han logrado el establecimiento en el mercado local.

- Creación, promoción y vinculación de las Mipymes a las redes interinstitucionales, que permita el establecimiento, intercambio y operativización oportuna de los programas y estrategias de transferencia tecnológica. 


\section{CONCLUSIONES}

Los emprendimientos son nuevas empresas que inician las personas por cuenta propia, con el propósito de percibir ingresos a través de la oferta de productos o servicios en diversos mercados, aspirando a posicionarse y ser competitivos en el sector que atienden. Las características que presentan estos negocios son similares, tales como: soñar en grande, la persistencia, asumir riesgos día a día, ser generadores de empleos y llegar a satisfacer las necesidades de un sector que esta desatendido o en auge, entre otras.

El emprendimiento y la innovación están estrechamente ligadas, ambas son agentes de cambio, a través de estas, se introducen en el mercado un nuevo o mejorado producto (bien o servicio). De igual manera, contribuyen al desarrollo económico de los países, a disminuir la pobreza y brinda bienestar a los ciudadanos, ya que, toman en cuenta las oportunidades y ponen en prácticas las ideas creativas, impulsando así la economía del país.

La innovación sigue siendo un reto para los emprendedores, se hace necesario incidir en la productividad de las operaciones, en la calidad del bien o servicio, desarrollar el capital humano, implementar tecnología en los procesos, para obtener una posición competitiva con respecto a sus competidores. De esta forma, la CTI, es uno de los motores centrales del crecimiento económico de mediano y largo plazo. Al implementar la CTI en los países, esto permite afrontar los desafíos con nuevos conocimientos y aplicaciones tecnológicas para optimizar la calidad de vida de los ciudadanos, de esta forma se resolverían algunos de los problemas de preocupación estratégica.

De acuerdo a los resultados de las investigaciones presentadas, a nivel de país, es evidente las debilidades en el tema de innovación; sobresaliendo los procesos productivos y de comercialización, afectando directamente la competitividad de estas empresas, la estabilidad y permanencia en el mercado.

Finalizando, Nicaragua cuenta con diversos programas enfocados en las Mipymes, dirigidos al fortalecimiento de estas empresas, los que son ejecutados por los diferentes Ministerios e Instituciones en todo el país, de igual manera, es visible los lineamientos y estrategias en el PNDH (2018-2021), cuyo propósito es atender las necesidades de transformación productiva y social del país.

\section{REFERENCIAS}

Centeno Ruiz, M. E., Gámez Velásquez, H. A., \& Mendoza Ocampo, D. C. (27 de Enero de 2020). Innovación en elproceso productivo de la Tabacalera Tamborde Esteli S.A,en el primer trimestre del año 2019. Recuperado el 19 de Junio de 2021, de https://repositorio.unan.edu.ni/13217/1/19950.pdf

CONICYT Presidencia de la República. (Agosto de 2010). Plan Nacional Ciencia, Tecnología e Innovación Nicaragua 2010-2013. Recuperado el 18 de Junio de 2021, de https://www.cepal.org/sites/default/ files/publication/files/32845/LCR2162.pdf

Díaz Galeano, G. N., Espinoza Calderón, R. d., \& Mairena Mejía, H. A. (16 de Diciembre de 2017). Innovación en el proceso productivo del sector tortillero del distrito II de la ciudad de Estelí, I semestre del año 2017. Recuperado el 19 de Junio de 2021, de https://repositorio.unan.edu.ni/9927/1/18943.pdf

Gutiérrez López, A., Rizo Ruíz , M., \& Rodríguez Flores, M. (Enero de 2016). “Caracterización de las PYMES 
del sector lácteo y su contribución económica a la ciudad de Estelí, en el periodo 2015.". Obtenido de https://repositorio.unan.edu.ni/2005/1/17355.pdf

Hernández Celis, D. (12 de Diciembre de 2016). Competitividad empresarial. Recuperado el 18 de Junio de 2021, de https://www.gestiopolis.com/competitividad-empresarial/

Herrera Guerra, C. E., \& Montoya Restrepo, L. (2013). El emprendedor: una aproximación a su definición y caracterización. Punto de vista, 10-22. Recuperado el 17 de Junio de 2021, de https://www.google. $\mathrm{com} / \mathrm{url}$ ?sa $=\mathrm{t} \& \mathrm{rct}=\mathrm{j} \& \mathrm{q}=\& \mathrm{esrc}=\mathrm{s} \&$ source $=$ web\&cd $=\& \mathrm{cad}=\mathrm{rja} \& u a c t=8 \& \mathrm{ved}=2 \mathrm{ahUKEwiJ} 15 \mathrm{vf2J}$ xAhVIiqwKHUy4CD8QFjAO egQIExAE\&url=https\%3A\%2F\%2Fdialnet.unirioja. es\%2Fdescarga\%2Farticulo\%2F4776922.pdf\&usg=AOvVaw0IUkHVnleUKY1i_a_Dmrru

Jiménez Coronado, A. M. (2016). Estrategia de competitividad y emprendimiento, una revisión de la literatura. Investigación e Innovación en Ingenierías, 104-126. Recuperado el 18 de Junio de 2021, de http://revistas.unisimon.edu.co/index.php/innovacioning/article/view/2492

Liévano, J., Vargas, P., Pico , B., \& Pérez, J. (2018). Importancia de La innovación para emprendedores de Micro Y Pequeñas Empresas Mexicanas del sector servicio en Villahermosa-Tabasco Y Mexicali-Baja California. Estudio Cuantitativo. ESJ AUGUST EDITION, Vol 14 No 22 (2018), 304-320. Recuperado el 17 de Junio de 2021, de https://eujournal.org/index.php/esj/article/view/11121

Molina Ycaza, D. E., \& Sánchez RioFrio, A. M. (2016). Factores de competitividad orientados a la pequeña y mediana empresa (PYME) en Latinoamérica: revisión de la literatura. Revista San Gregorio, 104111. doi:DOI: http://dx.doi.org/10.36097/rsan.v2i15.275

OECD/European Communities. (2005). Manual de Oslo: Guía para la recogida e interpretación de datos sobre innovacción. Recuperado el 17 de Junio de 2021, de http://www.itq.edu.mx/convocatorias/ manualdeoslo.pdf

Picado Juárez, A. M. (26 de Octubre de 2016). Estrategias competitivas utilizadas por las MIPYMES del sector servicio de comida buffet de la ciudad de Estelí en relación al marketing de servicio en el primer semestre 2016. Recuperado el 18 de Junio de 2021, de https://repositorio.unan.edu.ni/2882/

Picado Vanegas, A. J. (Diciembre de 2016). Innovación: El caso de la dinámica del subsector hortícola de la papa (Solanum tuberosum) y su índice de desarrollo en el norte de Nicaragua. Recuperado el 19 de Junio de 2021, de https://repositorio.unan.edu.ni/8268/1/6767.pdf

Somarriba Sandoval, F. L. (2015). ebuah.uah.es. Recuperado el 02 de Septiembre de 2020, de "Actividad Emprendedora en el Desarrollo Económico Local. Evidencias para el caso de Nicaragua y Municipio de León, 2000-2013".: https://ebuah.uah.es/dspace/handle/10017/23681

Urcuyo, R. (2012). Microfinanzas y Pequeñas y Medianas Empresas en Nicaragua. Managua: Banco Central de Nicaragua (BCN). Recuperado el 29 de Octubre de 2020, de https://pdfslide.tips/reader/f/ microfinanzas-y-pequenas-y-medianas-empresas-en-nicaragua 